\title{
Human umbilical cord multipotent mesenchymal stromal cells alleviate acute ischemia-reperfusion injury of spermatogenic cells via reducing inflammatory response and oxidative stress
}

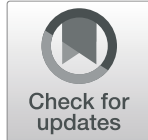

Liang Zhong ${ }^{1 \dagger}$, Mengbo Yang ${ }^{2 \dagger}$, Xiangyu Zou ${ }^{1 \dagger}$, Tao Du ${ }^{3}$, Huiming $\mathrm{Xu}^{2^{*}}$ and Jie Sun ${ }^{1 *}$

\begin{abstract}
Background: This study was designed to determine the effect of human umbilical cord multipotent mesenchymal stromal cells (hUC-MSC) on acute ischemia/reperfusion (I/R) injury of spermatogenic cells.

Method: The testicular I/R rat model was established through $720^{\circ}$ torsion for $1 \mathrm{~h}$. hUC-MSC were intravenously injected 10 min before detorsion. Injury severity of spermatogenic cells was estimated by Johnsen's score. The proliferating of recipient spermatogonia was measured by the immunostaining of antibodies against Ki67, and all germ cells were detected with DDX4 antibody. And recipient spermatogenesis was assessed by staining spermatozoa with lectin PNA. The levels of inflammatory factors were measured by real-time PCR. And the SelectinE expression, neutrophil infiltration in the testes was detected by immunostaining. Germ cells apoptosis was tested by TUNEL assay and western blot. Furthermore, the oxidative stress was tested by reactive oxidative species (ROS) levels. In vitro, the condition medium (CM) of hUC-MSC was used to culture human umbilical vein endothelial cells (HUVECs), so as to assess the paracrine effect of hUC-MSC on HUVECs. The protein chip was used to measure the relative concentration of the secretory proteins in the CM of hUC-MSC.

Result: hUC-MSC greatly alleviated the testicular injury induced by testis I/R. The levels of proinflammatory factors were downregulated by hUC-MSC in vivo and in vitro. Neutrophil infiltration, ROS, and germ cell apoptosis in testicular tissues were greatly reduced in the group of hUC-MSC. Paracrine factors secreted by hUC-MSC including growth factors, cytokines, and anti-inflammatory cytokine were rich.

(Continued on next page)
\end{abstract}

\footnotetext{
*Correspondence: quxuhm123@163.com; sunjie@scmc.com.cn

${ }^{\dagger}$ Liang Zhong, Mengbo Yang and Xiangyu Zou contributed equally to this work.

${ }^{2}$ State Key Laboratory of Oncogenes and Related Genes, Renji-Med X Clinical Stem Cell Research Center, Ren Ji Hospital, Shanghai Jiao Tong University School of Medicine, Shanghai 200127, China

'Department of Urology, Shanghai Children's Medical Center, Shanghai Jiao Tong University School of Medicine, Shanghai 200127, China

Full list of author information is available at the end of the article
}

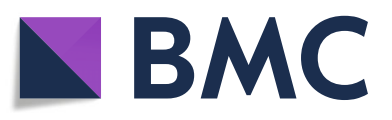

(๑) The Author(s). 2020 Open Access This article is licensed under a Creative Commons Attribution 4.0 International License, which permits use, sharing, adaptation, distribution and reproduction in any medium or format, as long as you give appropriate credit to the original author(s) and the source, provide a link to the Creative Commons licence, and indicate if changes were made. The images or other third party material in this article are included in the article's Creative Commons licence, unless indicated otherwise in a credit line to the material. If material is not included in the article's Creative Commons licence and your intended use is not permitted by statutory regulation or exceeds the permitted use, you will need to obtain permission directly from the copyright holder. To view a copy of this licence, visit http://creativecommons.org/licenses/by/4.0/. The Creative Commons Public Domain Dedication waiver (http://creativecommons.org/publicdomain/zero/1.0/) applies to the data made available in this article, unless otherwise stated in a credit line to the data. 


\begin{abstract}
(Continued from previous page)
Conclusion: This study demonstrated that intravenously injected hUC-MSC could protect the spermatogenic cells against I/R injury by reducing the inflammatory response, apoptosis, and acute oxidative injury. Paracrine mechanism of hUC-MSC may contribute to the protection of spermatogenic cells against I/R injury. Therefore, the present study provides a method for clinical treatment of attenuate I/R injury of spermatogenic cells.
\end{abstract}

Keywords: Stem cell, Ischemia-reperfusion injury, Spermatogenic cells, Inflammatory response, Oxidative stress

\section{Background}

Testicular torsion is a common type of urological emergency in adolescent males which usually leads to severe acute ischemia injury of the testis. Previous studies suggest that testicular torsion leads to male infertility because of testicular atrophy [1-3]. Testicular torsion in patients varies in time and degree, and it is commonly believed that the testicular salvage rate is $90 \%$ if spermatic cord detorsion is performed within $6 \mathrm{~h}$ from the onset of torsion [4]. But detorsion operation itself leads to ischemia/reperfusion (I/R) injury when the blood flow returns to normal. Many cellular and molecular mechanisms are investigated in testicular I/R injury. Inflammatory cascades are the most important pathological mechanism in I/R injury [5]. Apoptotic pathways are activated by inflammation which leads to germ-cellspecific apoptosis [6]. In addition, the production of ROS during I/R also causes DNA damage, endothelial damage, and germinal cell apoptosis [7]. Numerous pharmacological agents have been used as supportive therapy to prevent the adverse effects of I/R injury, including antioxidants, hormones, vitamins, and plant extracts [8]. But most of the agents only targeted to eliminate ROS which is less efficient.

MSCs have been reported to exert therapeutic effects by immunoregulation and suppression of oxidative stress in several diseases [9]. A study reported that MSCs transplanted by local injection into $\mathrm{I} / \mathrm{R}$ injured testis played a protective effect on germ cells [10]. However, local injection of MSCs may aggravate the intracompartmental pressure of testis, resulting in an occlusion of the microvascular bed that feeds the testicular lobules $[11,12]$. On the other hand, intravenous injection might be an appropriate transplantation method for MSCs, which can avoid these risks. Our previous study showed that in $I / R$ injury of the kidney model, intravenous mesenchymal stem cells were mainly blocked in the lung and still exhibited a great protective effect on the kidney $[13,14]$. In this study, we explore whether transvenous hUC-MSC attenuate acute I/R injury of spermatogenic cells.

\section{Methods}

\section{Isolation and culture of hUC-MSC}

hUC-MSCs were prepared and identified as described in our previous study [14]. In brief, the umbilical cord was obtained from women undergoing cesarean sections. Women gave informed consent for the collection of their umbilical cord. The collection and subsequent use of the umbilical cord were approved by the Institutional Ethical Review Committee of Shanghai Children's Medical Center, Shanghai Jiao Tong University School of Medicine. Umbilical cord tissues were cut and then attached to culture plates individually, followed by the addition of minimum essential medium $\alpha(\mathrm{MEM} \alpha)$ containing 10\% fetal bovine serum (FBS) (All from Life Technology). Approximately 12 days later, the colonies appeared and were cultured on new plastic plates for further expansion. The cells at the third to fifth passage were used in the following experiments.

\section{Testicular I/R rat model}

All works involving animals were approved by Institutional Ethical Review Committee of Shanghai Children's Medical Center, Shanghai Jiao Tong University School of Medicine. Adult male Sprague-Dawley rats weighing $180 \mathrm{~g}$ to $200 \mathrm{~g}$ were used. The testicular I/R rat model was established as described [15-17]. As shown in the Fig. S1, testicular I/R rat model was established through rotated $720^{\circ}$ in a clockwise direction fixing it to the scrotum with silk suture and maintained for $1 \mathrm{~h}$. Detorsion was performed by untwisting the testis. The success rate of the animal model was up to $80 \%$. Only the testes which return to red from purple or black after detorsion will be used in the further study. $10^{7}$ hUC-MSC in 0.5 $\mathrm{ml}$ PBS were injected into the tail vein $10 \mathrm{~min}$ before detorsion $(N=5)$, whereas control animals $(N=5)$ received $0.5 \mathrm{ml}$ PBS instead of the cells. Normal animals were used as the untreated group. The testes tissues were harvested at day 1 , day 3 , day 7 , and day 15 after intervention, respectively.

\section{Histopathology and PNA staining}

Fresh testicular tissues were washed with ice-cold PBS and kept at $-80^{\circ} \mathrm{C}$ until assay. Paraffin-embedded testis was sectioned at a thickness of $5 \mu \mathrm{m}$ and stained with hematoxylin and eosin (H\&E). Johnsen's score is a wellestablished method for evaluating spermatogenic function [18] and the severity of germ cell injury was quantified by Johnsen's score. Briefly, each section was given a score from 1 to 10 according to the following criteria: 
Score 10: Complete spermatogenesis with many spermatozoa. Germinal epithelium organized in a regular thickness leaving an open lumen. Score 9: Many spermatozoa present but germinal epithelium disorganized with marked sloughing or obliteration of the lumen. Score 8: Only few spermatozoa $(<5-10)$ present in the section. Score 7: No spermatozoa but many spermatids present. Score 6: No spermatozoa and only few spermatids $(<5-10)$ present. Score 5: No spermatozoa, no spermatids but several or many spermatocytes present. Score 4: Only few spermatocytes $(<5)$ and no spermatids or spermatozoa present. Score 3: Spermatogonia are the only germ cells present. Score 2: No germ cells but Sertoli cells are present. Score 1: No cells in the tubular section. The mean point value was from at least 10 seminiferous tubules.

Since PNA selectively binds to acrosome of sperms and can be used as a marker of sperm or haploid spermatid and further to evaluate integrity and numbers of sperms $[19,20]$. PNA staining was conducted according to the manufacturer's instructions. Generally, frozen slices were incubated with PNA (1:200) which conjugated with Alexa Fluor ${ }^{\text {rm }} 594$ (Life Technology) at room temperature for $30 \mathrm{~min}$, washed 3 times with PBS. Nuclei were stained with DAPI (Sigma-Aldrich) for $5 \mathrm{~min}$. Inverted fluorescence microscopy or confocal laser scanning microscopy was used to capture the image.

\section{Immunofluorescence staining}

For immunofluorescence analyses, ice frozen slices were blocked with 10\% donkey serum (Jackson, 017-000-121) for $0.5 \mathrm{~h}$ and then incubated with the primary antibodies: rabbit anti-MPO (Abcam, ab9535), rabbit anti-CD62E (Selectin-E, Abcam, ab18981), rabbit anti-Ki67 (Abcam, ab15580), rabbit anti-DDX4 (Abcam, ab13840) at $4{ }^{\circ} \mathrm{C}$ overnight. After washing with PBS three times, secondary antibodies, donkey anti-rabbit conjugated with Alexa 488(1:200, Life Technology), were incubated for $1 \mathrm{~h}$ at room temperature and then washed with PBS three times, nuclei were stained with DAPI. Inverted fluorescence microscopy was used to capture the image. The quantification results were evaluated in at least six representative visual fields for each group in a blinded manner by an experienced pathologist. Image-Pro Plus 6.0 (Media cybernetics, Silver Springs, MD, USA) was employed for image analysis.

\section{Assessment the levels of ROS in testis}

ROS is the indicator of oxidative stress level and can be used to evaluate the oxidative environment of testicular tissues. In situ visualization of ROS production was assessed by $2^{\prime}, 7^{\prime}$-dichlorodihydro fluorescein diacetate (DCFH-DA,10 $\mu \mathrm{mol} / \mathrm{L}$, Invitrogen, C6827) histochemistry. Nuclei were stained with DAPI before captured the image under microscopy. Fluorescence intensity of staining was measured by ImageJ. Six representative visual fields of each group were counted.

\section{The effect of hUC-MSC over the HUVECs in vitro}

We firstly collected the CM of hUC-MSC as follows: cells were cultured in complete medium up to $90 \%$ confluency, the cells were washed with PBS and changed with $\alpha$-MEM basic medium (Life Technology) for another $24 \mathrm{~h}$, and the medium was collected as hUC-MSCCM. Then HUVECs were cultured to $90 \%$ confluency in ECM complete medium containing ECM medium (Life Technology) with 10\% FBS and then changed with mixed medium which contained 50\% ECM complete medium and 50\% hUC-MSC-CM. ECM medium supplemented with 5\% FBS was used as a control medium. Ten nanograms/milliliter TNF- $\alpha$ was used to stimulate HUVECs for $24 \mathrm{~h}$. The HUVECs and cell supernatant were then collected to assess the expression of TNF- $\alpha$, IL-1 $\beta$, p-P65, and p-P38 and Selectin-E by real-time PCR and ELISA.

\section{RNA isolation and real-time quantitative PCR}

For RNA isolation, cells or testicular tissues were harvested and total RNA of samples was extracted using the TRIzol reagent and reverse transcribed into cDNA using the PrimeScript RT Reagent Kit (TAKARA, RR037A) according to the manufacturer's protocols. Real-time PCR was conducted with ChamQ Universal SYBR qPCR Master Mix (Vazyme, Q711-02) using Lightcycler 480 II (Roche) and normalized by the expression level of $\beta$ Actin. The information of primers was as follows:

$\beta$-Actin (human): forward sequence $\left(5^{\prime}-3^{\prime}\right)$ GGACAT CCGCAAAGACCTGTA, reverse sequence $\left(5^{\prime}-3^{\prime}\right)$ GCATCCTGTCGGCAATGC. TNF- $\alpha$ (human): forward sequence $\left(5^{\prime}-3^{\prime}\right)$ CCTCTCTCTAATCAGCCCTCTG, reverse sequence $\left(5^{\prime}-3^{\prime}\right)$ GAGGACCTGGGAGTAGAT GAG. IL-1 $\beta$ (human) forward sequence $\left(5^{\prime}-3^{\prime}\right)$ ATGA TGGCTTATTACAGTGGCAA, reverse sequence (5' 3') GTCGGAGATTCGTAGCTGGA. CD62E (Selectin$E$, human) forward sequence $\left(5^{\prime}-3^{\prime}\right)$, reverse sequence $\left(5^{\prime}-3^{\prime}\right)$ CCTTTGCTGACAATAAGCACTGG. $\beta$-Actin (rat): forward sequence $\left(5^{\prime}-3^{\prime}\right)$ TGTCACCAACTGGG ACGATA, reverse sequence $\left(5^{\prime}-3^{\prime}\right)$ GGGGTGTTGA AGGTCTCAAA. TNF- $\alpha$ (Rat):forward sequence (5'-3') CGCCACGAGCAGGAATGAGAAG, reverse sequence $\left(5^{\prime}-3^{\prime}\right)$ GCATGATCCGAGATGTGGAACTGG. IL$1 \beta$ (Rat): forward sequence $\left(5^{\prime}-3^{\prime}\right)$ CACACTAGCA GGTCGTCATCATCC, reverse sequence $\left(5^{\prime}-3^{\prime}\right)$ : ATCT CACAGCAGCATCTCGACAAG. CD62E (Selectin-E, Rat): forward sequence $\left(5^{\prime}-3^{\prime}\right)$ : GGTCTGCGATGCTG CCTACTTG, reverse sequence (5'-3'): GAAGTGAGGT TGCTGCCACAGAG. 


\section{Western blot}

Testicular tissues or HUVECs were lysed in RIPA (Thermo) with phosphatase inhibitor (Merck) and protease inhibitor (Merck) for $30 \mathrm{~min}$. Total protein concentration was measured by BCA protein assay kit (Thermo Fisher). The PVDF membranes transferred by proteins were blocked in 5\% nonfat powder milk and then incubated with primary antibodies against Selectin-E, P65, P38, $\beta$-Actin, Caspase 3, and $\beta$-tubulin (all from Cell Signaling Technology) overnight at $4{ }^{\circ} \mathrm{C}$. After washing with PBS three times, secondary antibody (Cell Signaling Technology) was incubated for $1 \mathrm{~h}$ at room temperature.

\section{ELISA test}

IL-1 $\beta$ concentration in the supernatant of HUVECs was detected by IL- $1 \beta$ ELISA kit (R\&D) according to the manufacturer's instructions.

\section{Protein chip detection of secretions}

The secretory protein in CM of hUC-MSC was accessed by human Antibody Array 507 protein chip (Raybiotech, AAH-BLG-507, glass slide). The procedure was done according to the manual of manufacture. Fluorescence signals were scanned with a GenePix 4000B (Axon Instruments, GenePix version 5.0). For each array, protein intensity values were background subtracted, scaled by the internal control, and floored at 1 unit. Human foreskin fibroblast cells (HEF) derived CM was used as control. HEF were isolated from Human discarded foreskin tissues and cultured as previously described [21]. The foreskin tissues were obtained from the circumcision upon the approval from the Institutional Ethical Review Committee of Shanghai Children's Medical Center, Shanghai Jiao Tong University School of Medicine. Written informed consent was obtained from the patient and guardian.

\section{Statistical analysis}

Data were presented as mean \pm SEM at least 3 experiments. The statistical analysis was conducted by $t$ test. $P$ value lower than 0.05 was considered significant. Statistical analysis was assessed by SPSS software 22.0. Quantification of fluorescence intensity was accessed by ImageJ.

\section{Results}

\section{hUC-MSC protect testes against I/R injury}

The histopathological images show that torsiondetorsion significantly damaged spermatogenic cells and reduced the Johnsen's score, especially at day 3 after detorsion (Fig. 1a, b; Fig. S2). But the MSC-treated testes had a marked improvement in Johnsen's score compared with that of control, suggesting that the hUC-MSC restore recipient spermatogenesis.
To further determine if hUC-MSC could protect recipient spermatogenesis, we analyzed recipient spermatogenesis by staining spermatozoa with lectin PNA conjugated with Alexa Fluor ${ }^{\text {tw }}$ 594, which selectively binds to acrosome of sperms and can be used as a sperm or haploid spermatid marker [20]. Figure 1c, d shows that seminiferous tubules of PNA-positive cells in the testes of hUC-MSC-treated rats were significantly increased compared to those of the control group. The data suggested that MSC has a protective effect on the survival of sperms.

As spermatogonia cells are crucial competent for the initiation of spermatogenesis, we next decided to determine whether hUC-MSC affected spermatogonia cell biology including testicular stem cells. We performed immunostaining of the testis tissues with Ki67 antibody (a marker reflecting the proliferating of spermatogonia) and DDX4 (a marker of germ cells including undifferentiated and differentiated spermatogonia). As shown in Fig. 1e, f, more Ki67- and DDX4-positive cells were present in the testes of the hUC-MSC group than those in the control testes. Moreover, DDX4-positive cells were evidently much more in the testes of the hUCMSC group at day 15 after transplantation. These data indicated that the hUC-MSC contributed to the recipient testicular stem cell niche and promoted the survival, proliferation, and differentiation of recipient spermatogonia cells.

\section{hUC-MSC reduce inflammatory response of spermatogenic cells induced by $\mathrm{I} / \mathrm{R}$}

Previous study reported that inflammatory response is the most important pathological mechanism during $I / R$ injury of testicular detorsion [5]. Therefore, we first tested the mRNA levels of inflammatory factors TNF- $\alpha$ and IL- $1 \beta$ of testicular tissues by real-time PCR. hUCMSC led to an obvious downregulation of mRNA levels of TNF- $\alpha$ and IL- $1 \beta$ at day 1 after detorsion compared to the control group (Fig. 2a). The data indicated that hUC-MSC reduced the expression levels of inflammatory factors of testis injured by $\mathrm{I} / \mathrm{R}$ at day 1 after detorsion. In addition, Selectin-E is an endothelial cell adhesion molecule which mediates the adhesion of neutrophils [22]; thus, we detected the level of Selectin-E of testicular tissues by real-time PCR and immunofluorescence. As shown in the Fig. 2b, the mRNA level of Selectin-E of testicular tissues in the group of hUC-MSC was significantly lower than that of control at day 1 after detorsion. The immunostaining analysis obtained a similar result on the expression of Selectin-E at day 1 after detorsion (Fig. 2c, d). However, the expression of Selectin-E is not upregulated 3 days after detorsion.

Next, we further measured the neutrophil infiltration. Because testis is an immune cell-free organ, neutrophil 


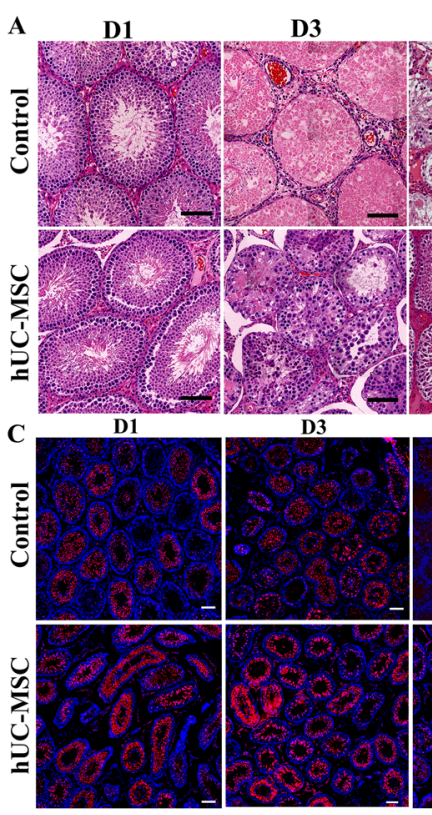

D7 D15 Normal

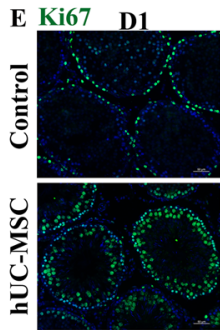

D3
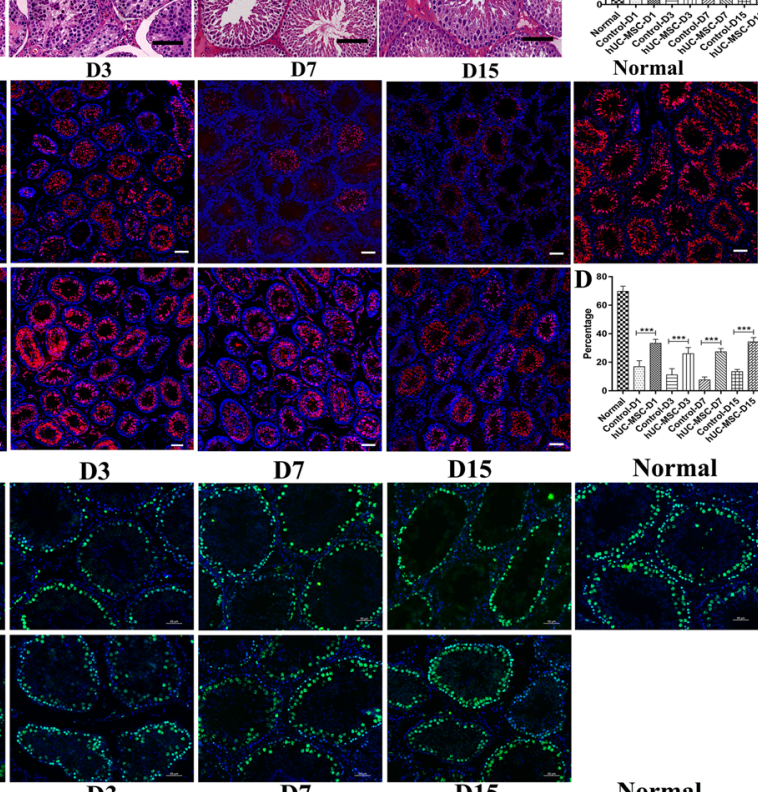

Normal
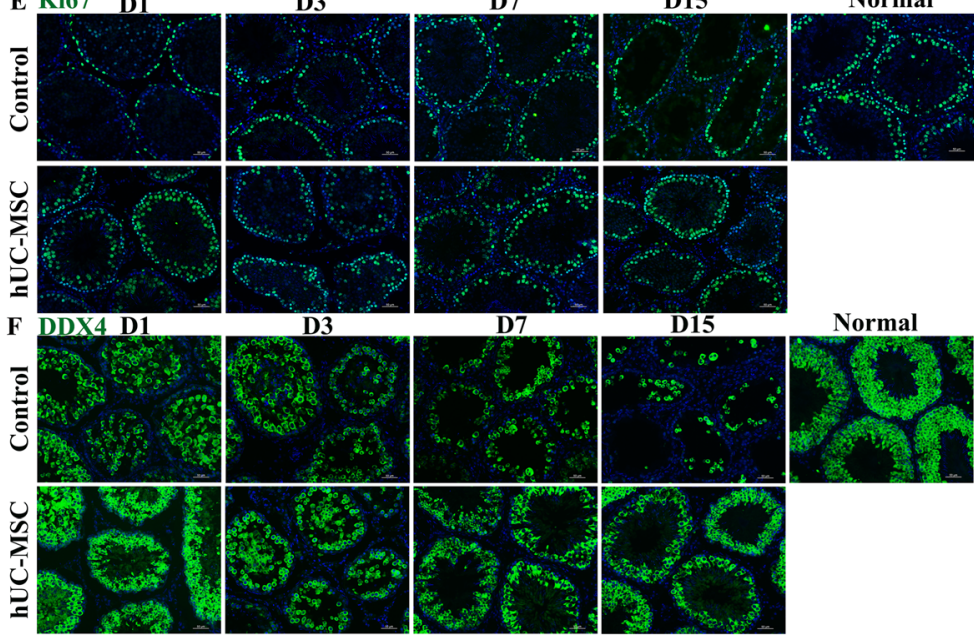

Normal

Fig. 1 hUC-MSC alleviated spermatogenic cells injury during testicular torsion and detorsion. a H\&E staining of rat testicular tissues at day 1 (D1), day 3 (D3), day 7 (D7), and day 15 (D15) after detorsion. The testes performed torsion and detorsion without hUC-MSC grafts were used as control. The normal group was untreated animals. Scale bars, $100 \mu \mathrm{m}$. b Johnsen's score was evaluated at indicated day after hUC-MSC treatment. c Staining with PNA. Scale bars, $200 \mu \mathrm{m}$. d Quantification of seminiferous tubules containing PNA-positive cells. Ten representative sections of the pattern of testes were counted. At least three rats were used in every group. Data were represented as mean $\pm \mathrm{SEM}$. ${ }^{*} P<0.05,{ }^{* *} P<0.01,{ }^{* * *} P<0.001$. e Immunostaining of rat seminiferous tubules at indicated day after torsion with proliferation marker Ki67. Scale bars, $50 \mu \mathrm{m}$. $\mathbf{f} I \mathrm{mmunostaining}$ of rat seminiferous tubules at indicated day after torsion with germ cell marker DDX4. Scale bars, $50 \mu \mathrm{m}$

infiltration may be the first response during the process of testicular I/R injury during torsion and detorsion operation; we tested the levels of MPO which was produced by neutrophils. As shown in Fig. 2e, f, the expression level of MPO was low at day 1 after detorsion, but high at day 3 after detorsion, and again returned to low level at day 7 and day 15 after detorsion, indicating that neutrophil infiltration happened at least 1 day after detorsion and reached its peak approximately at day 3. Furthermore, the level of MPO of testes in the hUC-MSC group was significantly lower than that of control. The results suggested that hUC-MSC remarkably reduced the levels of inflammatory factors and neutrophil infiltration of testes, resulting in a significant reduction of inflammatory response.

\section{hUC-MSC reduce the acute oxidative stress of spermatogenic cells induced by $\mathrm{I} / \mathrm{R}$ injury}

Previous research showed that ROS during I/R could cause DNA damage, endothelial damage, and germinal cell apoptosis [7]. In this study, we wonder if hUC-MSC could reduce the acute oxidative injury. Figure 3 shows 

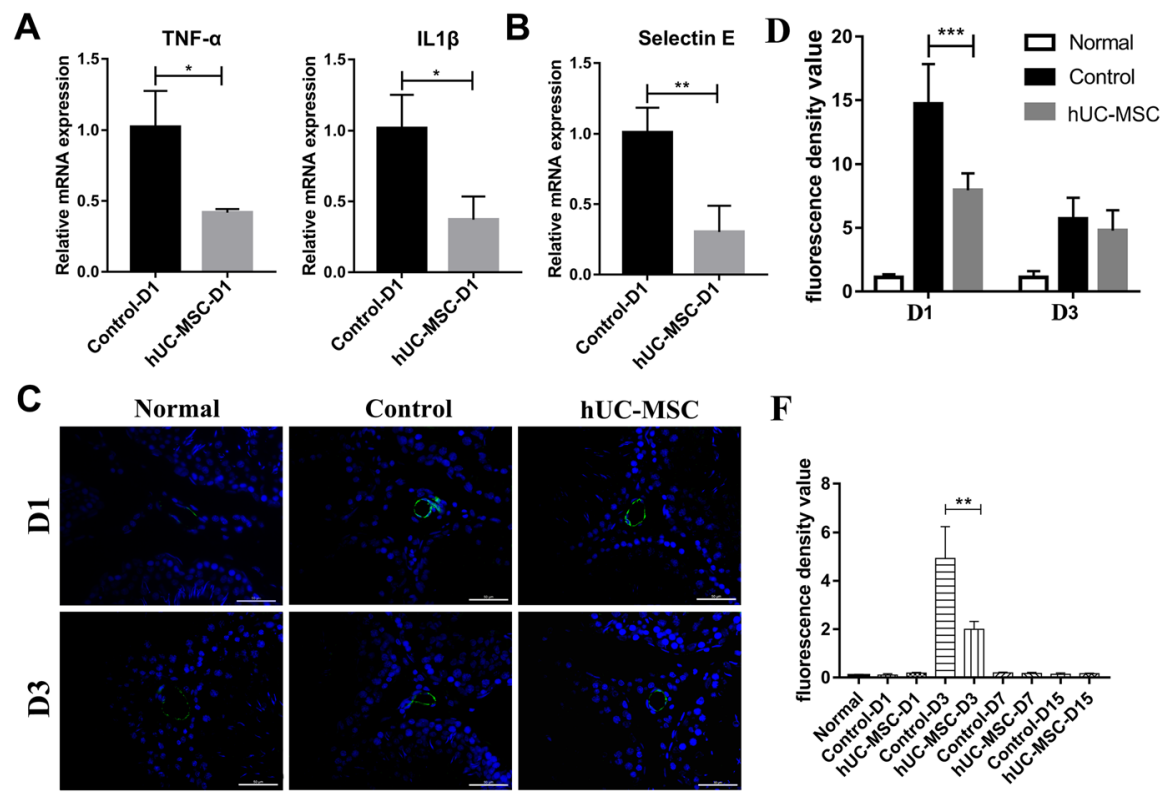

$\mathbf{E}$

D1

D3

D7
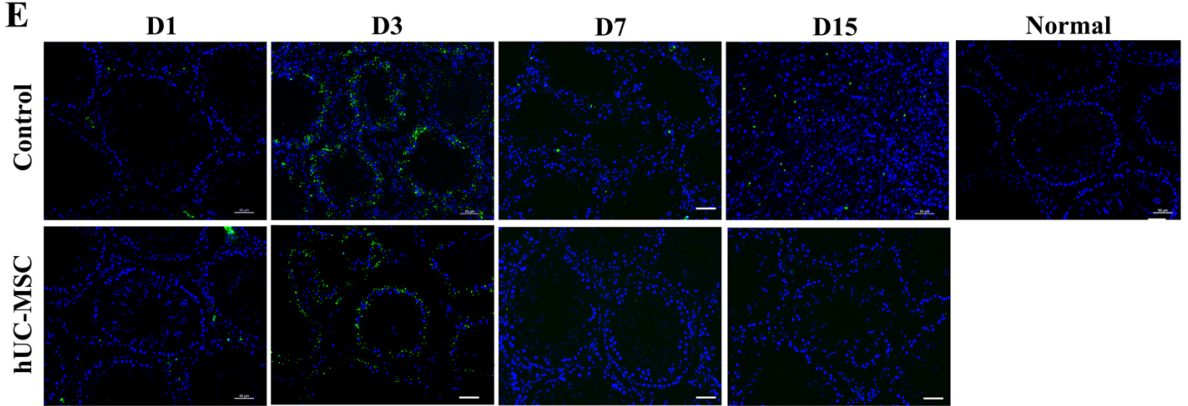

Fig. 2 hUC-MSC injection reduced inflammatory factor production, Selectin-E expression, and neutrophil infiltration of testicular tissues in rats subjected I/R injury. a Real-time PCR analysis of mRNA expression of TNF- $\alpha$ and IL-1 $\beta$ in testicular tissues. The level of mRNA in control testes without hUC-MSC grafts was set as 1. Data was collected from at least 3 rats. b Relative mRNA expression of Selectin-E in testicular tissues. $\mathbf{c}$ Immunofluorescence staining of testicular tissues of rats with Selectin-E antibody. Scale bars, $50 \mu \mathrm{m}$. d Quantification of fluorescence density of Selectin-E in every seminiferous tubule. At least 10 representative sections of testes were counted. At least three rats were used in every group. Data were represented as mean \pm SEM. E. Immunofluorescence staining of testicular tissues with MPO antibody. Scale bars, $50 \mu \mathrm{m}$. f Quantification of fluorescence density of MPO of every seminiferous tubule. At least 10 representative sections of testes were counted

that ROS were over-produced during I/R injury of testes, but the ROS level of testicular tissues in hUC-MSCtreated group was evidently lower than that of control at day 1 to day 15 after detorsion. The above results indicated that hUC-MSC significantly reduced ROS levels during I/R injury of spermatogenic cells.

\section{hUC-MSC protect germ cells against apoptosis induced by I/R injury}

Testicular torsion can lead to germ cell apoptosis and apoptotic pathways are often activated by inflammation and ROS $[6,23]$. Next, to determine whether hUC-MSC could protect germ cells against torsion-induced apoptosis and restore spermatogenesis, TUNEL assay and western blot were performed. Immunofluorescence staining with apoptosis marker TUNEL showed that testicular torsion induced germ cell apoptosis especially at day 1 and day 3 after detorsion, while testes in the normal group were negative for TUNEL (Fig. 4a). However, a significant reduction of germ cell apoptosis appeared in the group of hUC-MSC than the control group at day 1 and day 3 after detorsion (Fig. 4a). Of note, the level of TUNEL was low 7 days later after detorsion in both the control and hUC-MSC group. And more, germ cells in the seminiferous tubes were fewer in the control group than those of the hUC-MSC group (Figs. 4a and 1f). The data revealed that the apoptotic germ cells were dead 7 days after detorsion. Additionally, western blot analysis showed that the level of cleaved caspase 3 was lower than that of the control group (Fig. 4b). Taken together, hUCMSC reduced germ cell apoptosis induced by I/R injury and promote spermatogenesis. 


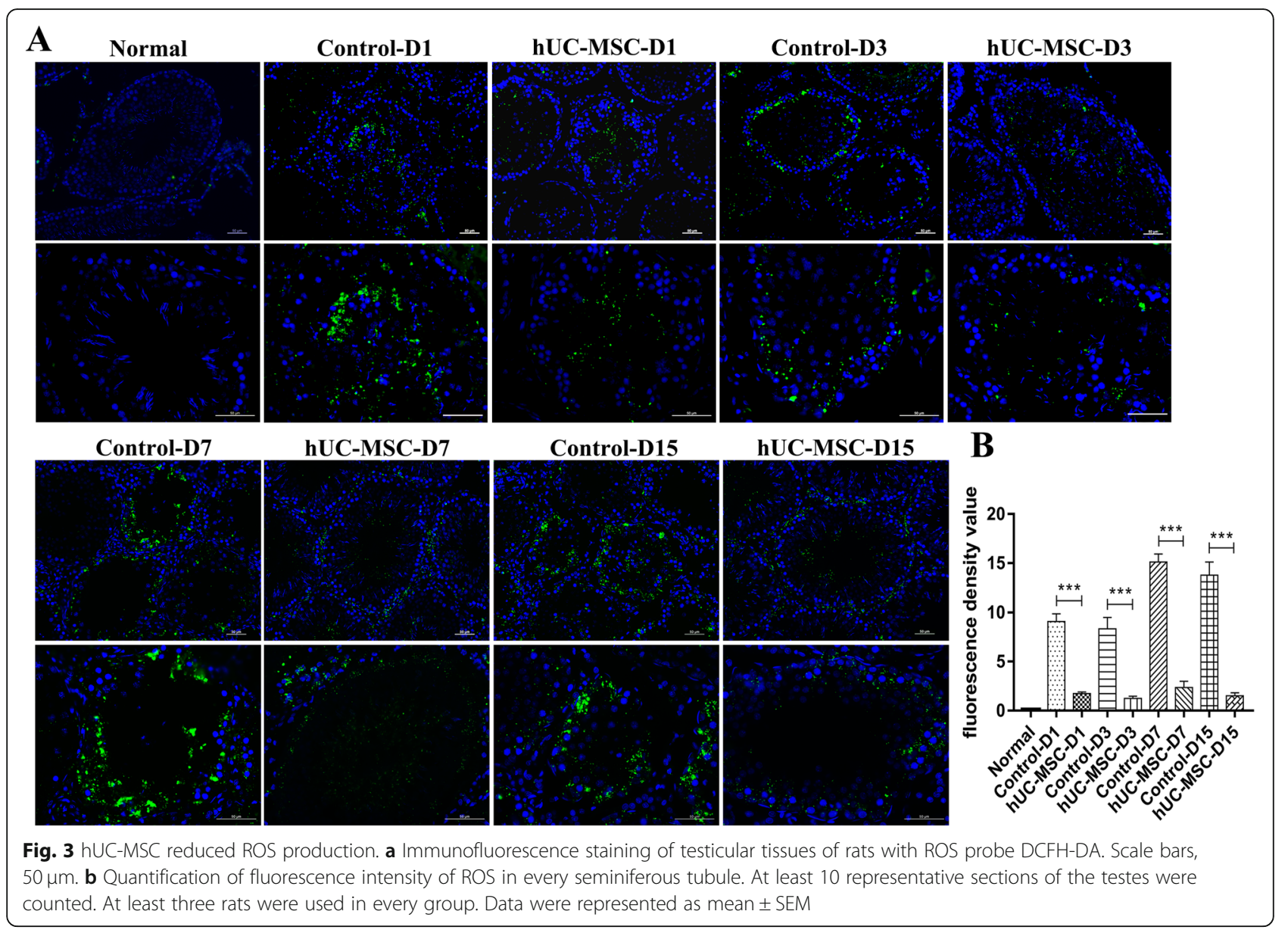

The CM of hUC-MSC reduce inflammatory response of HUVECs in vitro

Previous studies demonstrated that MSC could produce many paracrine factors contributing to the protection of cells against injury $[24,25]$. Thus, we wondered if the CM of hUC-MSC (hUC-MSC-CM) could reduce inflammatory response of HUVECs induced by TNF- $\alpha$. As shown in Fig. 5a, hUC-MSC-CM can reduce the mRNA levels of TNF- $\alpha$, IL-1 $\beta$, and Selectin-E of HUVECs stimulated by TNF- $\alpha$. And more, the concentration of IL- $1 \beta$ protein in the supernatant of HUVECs was also decreased in the hUC-MSC group (Fig. 5b). In addition, the protein level of Selectin-E was also decreased in the hUC-MSC-CM-treated group (Fig. 5c). Next, we would further determine if the inflammatory pathway was activated when HUVECs stimulated by TNF- $\alpha$. Western blot results showed that the expression of p-P65 and pP38 was evidently upregulated when HUVECs were stimulated by TNF- $\alpha$. However, hUC-MSC-CM could downregulate the level of p-P65 and p-P38 (Fig. 5d). These data suggested that hUC-MSC could reduce inflammatory response by their paracrine factors in vitro.

\section{Analysis of paracrine factors of hUC-MSC}

To determine what kinds of paracrine factors of hUCMSC-CM are beneficial to lesioned spermatogenic cells, we analyzed paracrine factors in both hUC-MSC-CM and HEF-CM (as a control) by the human antibody array kit against 507 soluble proteins. The data showed that the hUC-MSC-CM and HEF-CMs differed in the protein levels (Table S1). Anti-inflammatory cytokines IL1ra, IL10, IL13, TGF- $\beta 1$, and nutritive cytokines BDNF, GDNF, CNTF, HGF, FGF, EGF, and VEGF were richer in the hUC-MSC-CM compared to HEF-CM (Fig. 6a). Interestingly, HGF, which is well known to play a regulatory role of Selectin-E, was also richer in the hUC-MSCCM. Furthermore, KEGG pathway enrichment analysis revealed that several pathways including JAK-STAT, PI3K-Akt signaling, and MAPK signaling pathway are activated in the hUC-MSC-CM (Fig. 6b), which are crucial signaling pathways to germ cell proliferation and differentiation. Altogether, the results suggested that the anti-inflammatory factors and other growth factors could contribute to the protective effects of hUC-MSC against I/R injury of spermatogenic cells. 


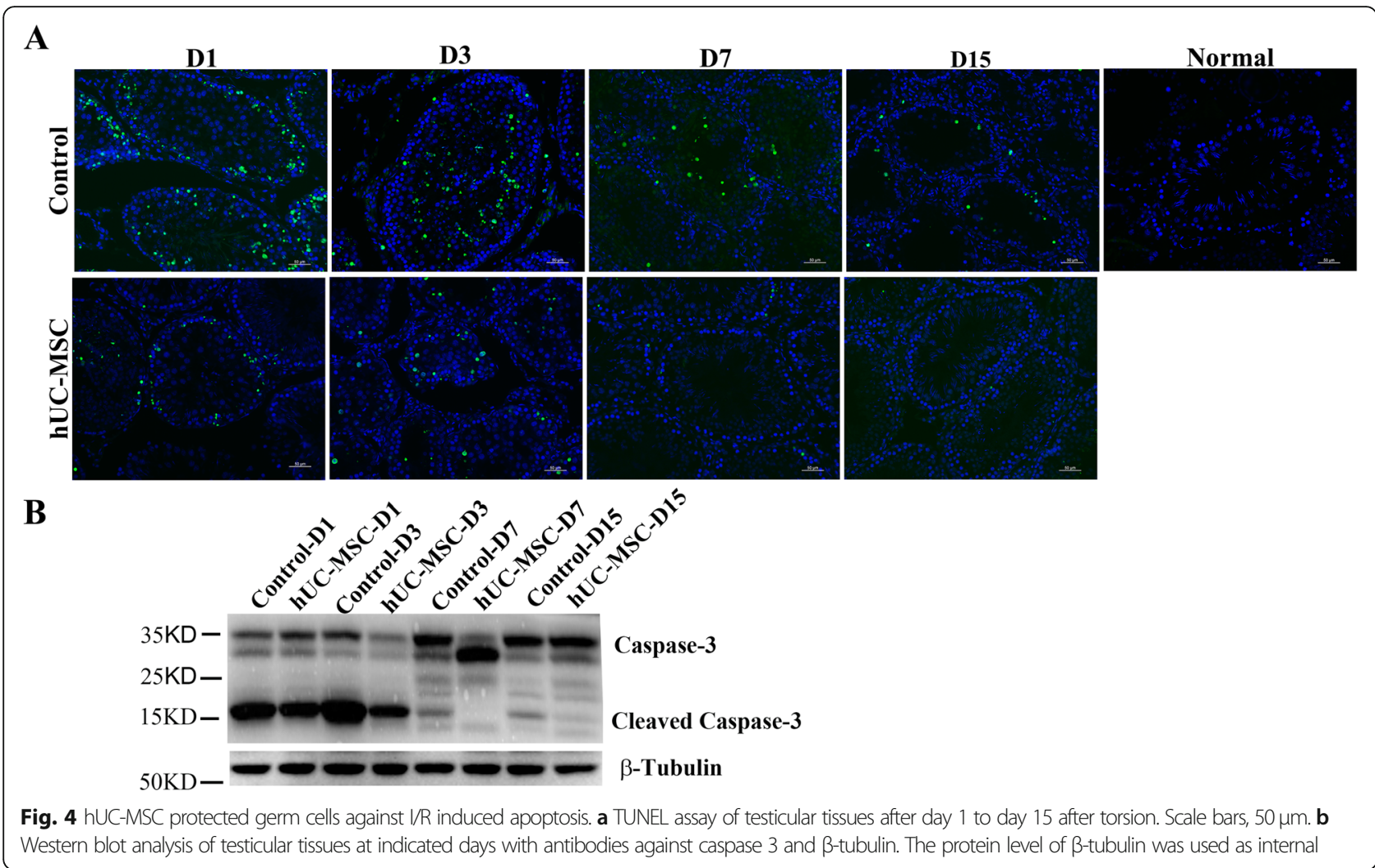

\section{Discussion}

Testicular torsion involving rotation of the testis and twisting of the spermatic cord will cause testicular atrophy. An immediate detorsion operation is required to prevent testicular ischemic necrosis within 4 to $8 \mathrm{~h}$ after torsion [26]. I/R injury during testicular torsion and detorsion operation of rat testis could result in a permanent loss of spermatogenesis despite the return of blood flow. Recently, MSCs are reported to be effective in attenuating myocardial $I / R$ injury in rats [27]. In this study, we found that hUC-MSC injected intravenously into rats subjected to testicular torsion and detorsion operation could attenuate I/R injury and promote the proliferation and differentiation of spermatogonia cells resulting in the survival and the regeneration of more spermatogenic cells and sperms (Fig. 1).

Many studies have demonstrated that MSCs have immunosuppressive activities owing to their paracrine effects and interactions with immune cells, which will subsequently reduce the level of TNF- $\alpha$ and IL-1 $\beta[28,29]$. Subsequently, TNF- $\alpha$ and IL-1 $\beta$ can activate JNK signaling pathway which leads to the expression of Selectin-E in endothelial cells and ultimately results in the recruitment of neutrophil [22, 30, 31]. In our study, the expression level of inflammatory factors TNF- $\alpha$ and IL- $1 \beta$ was upregulated during $\mathrm{I} / \mathrm{R}$ of the testis at day 1 after torsion, and more, hUC-MSC significantly decreased the expression level of inflammatory factors TNF- $\alpha$ and IL- $1 \beta$ at day 1 after torsion (Fig. 2a). However, the expression level of TNF- $\alpha$ and IL-1 $\beta$ did not increase compared to those of the normal group 3 days after detorsion (data not shown). Moreover, Selectin-E was upregulated during $I / R$ of the testis and hUC-MSC evidently reduced the expression level of Selectin-E at day 1 after torsion. Subsequently, the neutrophil infiltration began to appear mainly at day 3 after detorsion and then returned to a low level, and hUCMSC significantly reduced the neutrophil infiltration at day3 after detorsion (Fig. 2). Subsequently, the expression level of Selectin-E returned to a low level 3 days after testis detorsion (Fig. 2b-d). Consistent with our study, the downregulation of TNF- $\alpha$ and IL1 $\beta$ lead to the decline of Selectin-E gene expression [32-35]. In addition, neutrophil infiltration mainly appeared at day 3 after detorsion and then began to fall at day 7 and day 15 after detorsion evidenced by the expression of MPO was highest at day 3 after testis detorsion and became low again at day 7 and day 15 (Fig. 2e, f). The data indicated that inflammatory response during the testis I/R injury is an early pathological phenomenon and hUC-MSC could reduce the inflammatory response and promote the survival and regeneration of germ cells. In addition, in vitro hUC-MSC-CM had a similar inflammatory suppressive effect on HUVECs 


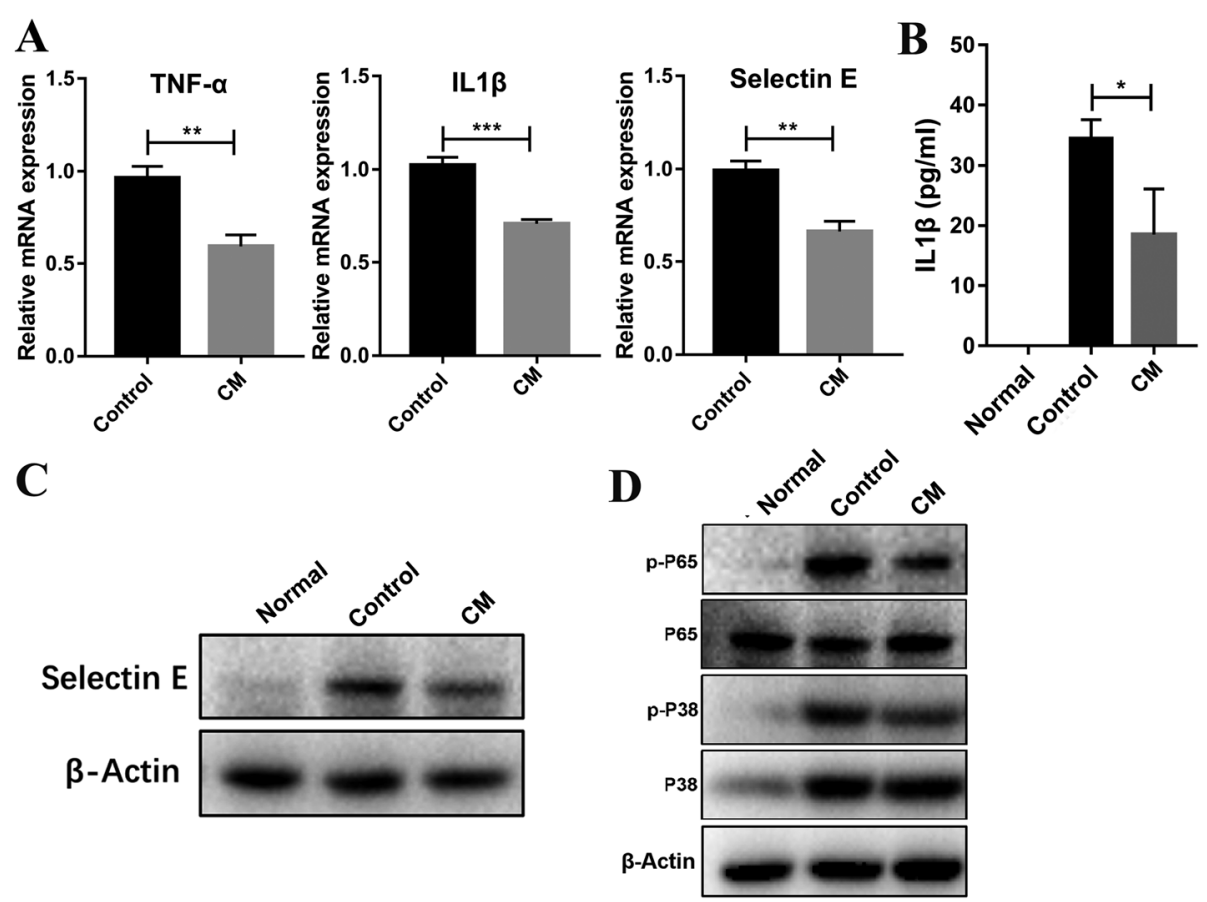

Fig. 5 hUC-MSC-CM inhibits inflammatory factors production and inflammatory pathway in HUVECs. HUVECs stimulated by $10 \mathrm{ng} / \mathrm{ml}$ TNF-a were cultured with or without hUC-MSC-CM. Twenty-four hours later, the cells were harvested for RNA extract. a Real-time PCR analysis of mRNA levels of TNF-a, IL-1 $\beta$, and Selectin-E of HUVECs. The level of mRNA in HUVECS was set as 1. Data was collected from at least 3 separated experiments. $\mathbf{b}$ ELISA analysis of IL-1 $\beta$ concentration in the supernatant of HUVECs. c Western blot analysis of Selectin-E expression of HUVECs with Selectin-E and $\beta$-actin antibodies. d Western blot analysis of P65 and P38 activation of HUVECs with antibodies against P-P65, P65, P-P38, P38, and $\beta$-actin. CM represents hUC-MSC-CM

stimulated by TNF- $\alpha$, evidenced by decreased levels of TNF- $\alpha$, IL-1 $\beta$, and Selectin-E of HUVECs and P65, P38 phosphorylation (Fig. 5). This result indicated that hUCMSC can reduce inflammatory response by paracrine factors.

As for molecular mechanism, many anti-inflammatory cytokines, such as IL-1ra, IL10, IL13, and TGF- $\beta 1$, were detected by protein chip and rich in the CM of hUCMSC (Table S1 and Fig. 6). Of note, IL-1ra, IL10, and IL13 are demonstrated to reduce inflammatory response [36-39]. Therefore, the anti-inflammatory cytokines may contribute to the immunosuppressive function of hUCMSC on I/R injury of spermatogenic cells. In addition, the HGF in the CM of hUC-MSC may play a role on the inhibition of the expression of Selectin-E. Previous studies revealed that HGF can inhibit neutrophil infiltration via the downregulation of Selectin-E on the endothelial cell surface, which suppresses ischemia-related injury in various organs [40-42]. Thus, HGF secreted by hUCMSC may downregulate Selectin-E to reduce neutrophil infiltration. In summary, hUC-MSC could reduce inflammatory response by secreting anti-inflammatory factors, HGF, and other factors.
Additionally, ROS is produced through normal metabolic reactions and have roles in the processes such as pathogen killing and cell signaling [43]. Overgeneration of ROS has been associated with I/R injury in different organs and testes are highly sensitive to ROS damage [44]. In this study, ROS was over-produced during I/R injury of testes at day 1 to day 15 after torsion (Fig. 3). But hUC-MSC could significantly reduce the level of ROS, indicating that hUC-MSC could decrease oxidative stress and oxidative injury during testis torsion and detorsion.

On the other hand, previous studies found that testis torsion could induce germ cell apoptosis. In the testis torsion and detorsion animal experiment, germ cell apoptosis was evident at day 1 and day 3 after torsion; however, it was reduced at day 7 and day 15 after torsion. The data revealed that testis torsion first induced germ cell apoptosis; subsequently, the apoptotic germ cells were dead, evidenced by fewer germ cells (DDX4positive cells) in the seminiferous tubules in the control group (Figs. 1 and 4a). Interestingly, hUC-MSC protected germ cells against torsion-induced apoptosis and restored spermatogenesis (Figs. 1 and 4a). 


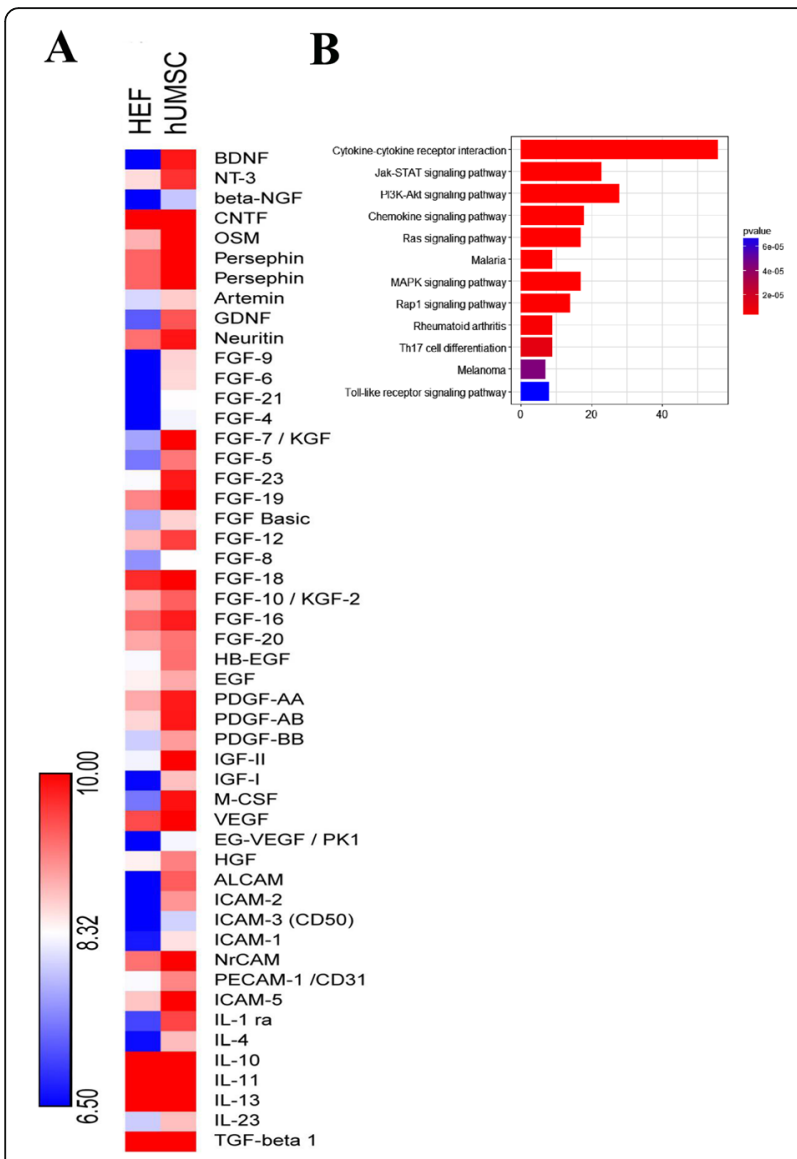

Fig. 6 Normalized array data of the 507 proteins were analyzed by SAM to detect a difference in their concentrations between the hUC-MSC-CM and HEF-CM. a The relative concentrations of the neurotrophic factors, growth factors, cell adhesion molecules, and anti-inflammatory factors that obtained a significant score ( $q$ value $<0.001 \%)$ are shown in a "heatmap." Low concentrations are shown in blue, medium concentrations in white and high concentrations in red. Also, see Table S1. b KEGG pathway analysis of the soluble factors in the CM of hUC-MSC and hEF. Enriched pathways in the CM of hUC-MSCS that obtained a significant score ( $P$ value $<0.05)$. HEF represents hEF-CM. hUMSC presents hUC-MSC-CM

Stem cells can be transplanted through local injection or intravenous injection. Though local injection can bypass the blood-testis barrier and directly affect the testis, it is not a good treatment option in clinical practice. The potential risk of compartment syndrome by local injection has limited its clinical application [11, 12]. In our previous study of $I / R$ injury of the kidney, intravenous stem cells still conducted a great protective effect though they were blocked in the lung [13]. In the present study, hUC-MSC possibly played a suppressive role on inflammatory response and decline of acute oxidative injury via the paracrine factors. Interestingly, we found a large number of nutritive cytokines and anti-inflammatory factors in the CM of hUC-MSC. They may be able to enter the testis via the circulation and reduce inflammatory response and ROS and support the survival and growth of testicular cells during I/R injury of testes.

\section{Conclusions}

In summary, this study demonstrated that hUC-MSC could protect the spermatogenic cells against $I / R$ injury. And more, hUC-MSC could reduce inflammatory response evidenced by downregulating the expression of inflammatory factor and infiltration of neutrophils. Furthermore, hUC-MSC ameliorated acute oxidative injury by reducing the ROS level. Besides, hUC-MSC protected germ cell apoptosis induced by $\mathrm{I} / \mathrm{R}$ injury and promoted spermatogenesis. Paracrine factors secreted by MSC may be a main mechanism. Therefore, the present study provides a method for clinical treatment of I/R injury during testes detorsion operation to facilitate the spermatogenesis.

\section{Supplementary information}

Supplementary information accompanies this paper at https://doi.org/10. 1186/s13287-020-01813-5.

Additional file 1: Figure S1. Presentation of testicular $I / R$ rat model method. The testicular I/R rat model was established through 720-degree torsion for $1 \mathrm{~h}$ and the testes became purple, then the testes returned to red from purple or black after detorsion. hUC-MSC were intravenously injected $10 \mathrm{~min}$ before detorsion. Only the testes which return to red after detorsion will be used in the further study.

Additional file 2: Figure S2. hUC-MSC alleviated spermatogenic cells injury during testicular torsion and detorsion. (A, B) The images of the entire section of mouse testes at indicted time after torsion by $H \& E$ staining(A) and PNA staining (B). Scale bars:2000 $\mu \mathrm{m}$.

Additional file 3: Table S1. A table of normalized fluorescence signal intensity of neurotrophic factors, growth factors, cell adhesion molecules (CAM) and anti-inflammatory factors in the HEF-CM or hUC-MSC-CM and ratio of them.

\section{Abbreviations}

hUC-MSC: Human umbilical cord mesenchymal stromal cells; I/R: Ischemia/ reperfusion; ROS: Reactive oxidative species; DCFH-DA: 2',7'-Dichlorodihydro fluorescein diacetate; CM: Condition medium; HUVECs: Human umbilical vein endothelial cells; MEMa: Minimum essential medium a; FBS: Fetal bovine serum; H\&E: Hematoxylin and eosin; MPO: Myeloperoxidase; HEF: Human foreskin fibroblast cells

\section{Acknowledgements}

Not applicable

\section{Authors' contributions}

Liang Zhong, Mengbo Yang, Xiangyu Zou, Tao Du, Huiming Xu, and Jie Sun were all involved in the study design, data acquisition, and analysis. The authors read and approved the final manuscript.

\section{Funding}

This study is supported by the National Natural Science Foundation of China 81601267 and 81871149, Foundation of Shanghai Jiaotong University ZH2018ZDB07.

\section{Availability of data and materials}

All data generated or analyzed during this study are included in this published article and its supplementary information files. 


\section{Ethics approval and consent to participate}

All works involving animals, the collection of the umbilical cord and foreskin tissues, were approved by the Institutional Ethical Review Committee of Shanghai Children's Medical Center, Shanghai Jiao Tong University School of Medicine. Written informed consent was obtained from individual participants or guardian.

\section{Consent for publication}

Not applicable

\section{Competing interests}

The authors declare that they have no competing interests.

\section{Author details}

'Department of Urology, Shanghai Children's Medical Center, Shanghai Jiao Tong University School of Medicine, Shanghai 200127, China. ${ }^{2}$ State Key Laboratory of Oncogenes and Related Genes, Renji-Med X Clinical Stem Cell Research Center, Ren Ji Hospital, Shanghai Jiao Tong University School of Medicine, Shanghai 200127, China. ${ }^{3}$ Department of Urology, Henan Provincial People's Hospital, Zhengzhou City 450003, China.

Received: 2 March 2020 Revised: 17 June 2020

\section{Accepted: 6 July 2020 Published online: 17 July 2020}

\section{References}

1. Anderson MJ, Dunn JK, Lipshultz LI, Coburn M. Semen quality and endocrine parameters after acute testicular torsion. J Urol. 1992;147(6):1545-50.

2. Arap MA, Vicentini FC, Cocuzza M, Hallak J, Athayde K, Lucon AM, et al. Late hormonal levels, semen parameters, and presence of antisperm antibodies in patients treated for testicular torsion. J Androl. 2007;28(4):528-32. https:// doi.org/10.2164/jandrol.106.002097 Epub 2007 Feb 7.

3. Jacobsen FM, Rudlang TM, Fode M, Ostergren PB, Sonksen J, Ohl DA, et al. The impact of testicular torsion on testicular function. World J Mens Health. 2019;10(37):190037.

4. Ringdahl E, Teague L. Testicular torsion. Am Fam Physician. 2006;74(10): 1739-43.

5. Karaguzel E, Kadihasanoglu M, Kutlu O. Mechanisms of testicular torsion and potential protective agents. Nat Rev Urol. 2014;11(7):391-9. https://doi.org/ 10.1038/nrurol.2014.135 Epub Jun 17.

6. Turner TT, Bang HJ, Lysiak JL. The molecular pathology of experimental testicular torsion suggests adjunct therapy to surgical repair. J Urol. 2004; 172(6 Pt 2):2574-8

7. Üstün H, Akgül KT, Ayyıldız A, Yağmurdur H, Nuhoğlu B, Karagüzel E, et al. Effect of phospodiesterase 5 inhibitors on apoptosis and nitric oxide synthases in testis torsion: an experimental study. Pediatr Surg Int. 2007;24(2):205-11.

8. Shimizu S, Tsounapi P, Dimitriadis F, Higashi Y, Shimizu T, Saito M. Testicular torsion-detorsion and potential therapeutic treatments: a possible role for ischemic postconditioning. Int J Urol. 2016;23(6):454-63. https://doi.org/10 1111/iju.13110 Epub 2016 May 23.

9. Uccelli A, Moretta L, Pistoia V. Mesenchymal stem cells in health and disease. Nat Rev Immunol. 2008;8(9):726-36. https://doi.org/10.1038/nri2395.

10. Hsiao CH, Ji AT, Chang CC, Cheng CJ, Lee LM, Ho JH. Local injection of mesenchymal stem cells protects testicular torsion-induced germ cell injury. Stem Cell Res Ther. 2015;6:113. https://doi.org/10.1186/s13287-015-0079-0.

11. Watson MJ, Bartkowski DP, Nelson NC. Intracompartmental pressure as a predictor of intratesticular blood flow: a rat model. J Urol. 2015;193(6):20627. https://doi.org/10.1016/j.juro.2014.12.018 Epub Dec 10.

12. Moritoki Y, Kojima Y, Mizuno K, Kamisawa H, Kohri K, Hayashi Y. Intratesticular pressure after testicular torsion as a predictor of subsequent spermatogenesis: a rat model. BJU Int. 2012;109(3):466-70. https://doi.org/ 10.1111/j.464-410X.2011.10279.x discussion 70 Epub 2011 May 23.

13. Du T, Cheng J, Zhong L, Zhao XF, Zhu J, Zhu YJ, et al. The alleviation of acute and chronic kidney injury by human Wharton's jelly-derived mesenchymal stromal cells triggered by ischemia-reperfusion injury via an endocrine mechanism. Cytotherapy. 2012;14(10):1215-27.

14. Du T, Zou XY, Cheng J, Wu S, Zhong L, Ju GQ, et al. Human Wharton's jellyderived mesenchymal stromal cells reduce renal fibrosis through induction of native and foreign hepatocyte growth factor synthesis in injured tubular epithelial cells. Stem Cell Res Ther. 2013;4(3):59.

15. Ghasemnejad-Berenji M, Ghazi-Khansari M, Pashapour S, Jafari A, Yazdani I, Ghasemnejad-Berenji $\mathrm{H}$, et al. Synergistic effect of rapamycin and metformin against germ cell apoptosis and oxidative stress after testicular torsion/ detorsion-induced ischemia/reperfusion in rats. Biomed Pharmacother. 2018, 105:645-51. https://doi.org/10.1016/j.biopha.2018.06.012 Epub Jun 11.

16. Payabvash S, Kiumehr S, Tavangar SM, Dehpour AR. Ethyl pyruvate reduces germ cell-specific apoptosis and oxidative stress in rat model of testicular torsion/detorsion. J Pediatr Surg. 2008;43(4):705-12.

17. Turner TT, Tung KS, Tomomasa H, Wilson LW. Acute testicular ischemia results in germ cell-specific apoptosis in the rat. Biol Reprod. 1997;57(6): 1267-74 doi: 10.095/biolreprod57.6.

18. Johnsen SG. Testicular biopsy score count--a method for registration of spermatogenesis in human testes: normal values and results in 335 hypogonadal males. Hormones. 1970;1(1):2-25.

19. Aviles M, Castells MT, Martinez-Menarguez JA, Abascal I, Ballesta J. Localization of penultimate carbohydrate residues in zona pellucida and acrosomes by means of lectin cytochemistry and enzymatic treatments. Histochem J. 1997;29(8):583-92. https://doi.org/10.1023/a:1026432211012.

20. Szasz F, Sirivaidyapong S, Cheng FP, Voorhout WF, Marks A, Colenbrander B, et al. Detection of calcium ionophore induced membrane changes in dog sperm as a simple method to predict the cryopreservability of dog semen. Mol Reprod Dev. 2000;55(3):289-98. https://doi.org/10.1002/(SICl)982795(200003)55:3<289::AID-MRD7>3.0.CO;2-K.

21. Li C, Yu H, Ma Y, Shi G, Jiang J, Gu J, et al. Germline-competent mouseinduced pluripotent stem cell lines generated on human fibroblasts without exogenous leukemia inhibitory factor. PLoS One. 2009;4(8):e6724. https://doi. org/10.1371/journal.pone.0006724.

22. Lysiak JJ, Turner SD, Nguyen QA, Singbartl K, Ley K, Turner TT. Essential role of neutrophils in germ cell-specific apoptosis following ischemia/ reperfusion injury of the mouse testis. Biol Reprod. 2001;65(3):718-25.

23. Ustun H, Akgul KT, Ayyildiz A, Yagmurdur H, Nuhoglu B, Karaguzel E, et al. Effect of phospodiesterase 5 inhibitors on apoptosis and nitric oxide synthases in testis torsion: an experimental study. Pediatr Surg Int. 2008; 24(2):205-11. https://doi.org/10.1007/s00383-007-2058-8 Epub 2007 Nov 6

24. Torrente D, Avila MF, Cabezas R, Morales L, Gonzalez J, Samudio I, et al. Paracrine factors of human mesenchymal stem cells increase wound closure and reduce reactive oxygen species production in a traumatic brain injury in vitro model. Human Experimental Toxicol. 2014;33(7):673-84.

25. Pianta S, Bonassi Signoroni P, Muradore I, Rodrigues MF, Rossi D, Silini A et al. Amniotic membrane mesenchymal cells-derived factors skew T cell polarization toward Treg and downregulate Th1 and Th17 cells subsets. Stem Cell Rev Rep. 2015;11(3):394-407.

26. Bartsch G, Frank S, Marberger H, Mikuz G. Testicular torsion: late results with special regard to fertility and endocrine function. J Urol. 1980;124(3):375-8.

27. Crisostomo PR, Wang M, Wairiuko GM, Morrell ED, Terrell AM, Seshadri P, et al. High passage number of stem cells adversely affects stem cell activation and myocardial protection. Shock. 2006;26(6):575-80.

28. Sun Y, Kong W, Huang S, Shi B, Zhang H, Chen W, et al. Comparable therapeutic potential of umbilical cord mesenchymal stem cells in collageninduced arthritis to TNF inhibitor or anti-CD20 treatment. Clin Exp Rheumatol. 2017;35(2):288-95 Epub 2017 Jan 15.

29. Liu C, Zhang H, Tang X, Feng R, Yao G, Chen W, et al. Mesenchymal stem cells promote the osteogenesis in collagen-induced arthritic mice through the inhibition of TNF-alpha. Stem Cells Int. 2018;2018:4069032. https://doi. org/10.1155/2018/4069032 eCollection 2018.

30. Lysiak JJ. The role of tumor necrosis factor-alpha and interleukin-1 in the mammalian testis and their involvement in testicular torsion and autoimmune orchitis. Reprod Biol Endocrinol. 2004;2:9. https://doi.org/10. 1186/477-7827-2-9.

31. Brenner D, Blaser H, Mak TW. Regulation of tumour necrosis factor signalling: live or let die. Nat Rev Immunol. 2015;15(6):362-74. https://doi. org/10.1038/nri3834

32. Kim DH, Lee SM, Lee YJ, Yoon JJ, Tan R, Yu YC, et al. Effect of Paeotang on tumor necrosis factor alpha-induced vascular inflammation in human umbilical vein endothelial cells. Chin J Integr Med. 2017;24(10):017-2759.

33. Nallasamy P, Si H, Babu PV, Pan D, Fu Y, Brooke EA, et al. Sulforaphane reduces vascular inflammation in mice and prevents TNF-alpha-induced monocyte adhesion to primary endothelial cells through interfering with the NF-kappaB pathway. J Nutr Biochem. 2014;25(8):824-33. https://doi.org/ 10.1016/j.jnutbio.2014.03.011 Epub Apr 4.

34. Lin CW, Chen LJ, Lee PL, Lee Cl, Lin JC, Chiu JJ. The inhibition of TNF-alphainduced E-selectin expression in endothelial cells via the JNK/NF-kappaB pathways by highly $\mathrm{N}$-acetylated chitooligosaccharides. Biomaterials. 2007; 28(7):1355-66 doi: 10.016/j.biomaterials.2006.11.006. Epub Nov 28. 
35. Zhao B, Stavchansky SA, Bowden RA, Bowman PD. Effect of interleukin1 beta and tumor necrosis factor-alpha on gene expression in human endothelial cells. Am J Physiol Cell Physiol. 2003;284(6):C1577-83 doi: 10. 152/ajpcell.00243.2002. Epub 2003 Jan 29.

36. Ortiz LA, Dutreil M, Fattman C, Pandey AC, Torres G, Go K, et al. Interleukin 1 receptor antagonist mediates the antiinflammatory and antifibrotic effect of mesenchymal stem cells during lung injury. Proc Natl Acad Sci U S A. 2007; 104(26):11002-7.

37. Schiff MH. Role of interleukin 1 and interleukin 1 receptor antagonist in the mediation of rheumatoid arthritis. Ann Rheum Dis. 2000;59(Suppl 1):i103-8.

38. Wang J, Ren $H$, Yuan X, Ma H, Shi X, Ding Y. Interleukin-10 secreted by mesenchymal stem cells attenuates acute liver failure through inhibiting pyroptosis. Hepatol Res. 2018;48(3):E194-202

39. Woods JM, Amin MA, Katschke KJ Jr, Volin MV, Ruth JH, Connors MA, et al. Interleukin-13 gene therapy reduces inflammation, vascularization, and bony destruction in rat adjuvant-induced arthritis. Hum Gene Ther. 2002;13(3): 381-93.

40. Mizuno S, Nakamura T. Prevention of neutrophil extravasation by hepatocyte growth factor leads to attenuations of tubular apoptosis and renal dysfunction in mouse ischemic kidneys. Am J Pathol. 2005;166(6): 1895-905 doi: 10.016/50002-9440(10)62498-4.

41. Makondo K, Kimura K, Kitamura T, Yamaji D, Dong Jung B, Shibata H, et al. Hepatocyte growth factor/scatter factor suppresses TNF-alpha-induced Eselectin expression in human umbilical vein endothelial cells. Biochim Biophys Acta. 2004;1644(1):9-15. https://doi.org/10.1016/j.bbamcr.2003.10.006.

42. Gong R, Rifai A, Dworkin LD. Hepatocyte growth factor suppresses acute renal inflammation by inhibition of endothelial E-selectin. Kidney Int. 2006; 69(7):1166-74.

43. Poljsak B, Suput D, Milisav I. Achieving the balance between ROS and antioxidants: when to use the synthetic antioxidants. Oxidative Med Cell Longevity. 2013;2013:956792. https://doi.org/10.1155/2013/956792 Epub 2013 Apr 29.

44. Filho DW, Torres MA, Bordin AL, Crezcynski-Pasa TB, Boveris A. Spermatic cord torsion, reactive oxygen and nitrogen species and ischemiareperfusion injury. Mol Asp Med. 2004;25(1-2):199-210. https://doi.org/10. 1016/.jmam.2004.02.020.

\section{Publisher's Note}

Springer Nature remains neutral with regard to jurisdictional claims in published maps and institutional affiliations.

Ready to submit your research? Choose BMC and benefit from:

- fast, convenient online submission

- thorough peer review by experienced researchers in your field

- rapid publication on acceptance

- support for research data, including large and complex data types

- gold Open Access which fosters wider collaboration and increased citations

- maximum visibility for your research: over $100 \mathrm{M}$ website views per year

At $\mathrm{BMC}$, research is always in progress.

Learn more biomedcentral.com/submissions 